\title{
Zur Problematik sozialpsychologiseher Interpretation des Verhaltens von Kindern in Zwei-Personen-Spielen
}

\author{
Von W. Hommers und V. Trempler
}

Mit 1 Abbildung

\section{Problemstellung}

Kooperatives Verhalten wurde unter den Bedingungen von Zwei-Personen-Nichtnullsummen-Spielen näher untersucht. In den bekanntesten Spielen dieser Spielklasse, dem „Prisoner's Dilemma Game“ (PDG) und dem „Maximizing Differences Game" (MDG) [12] entscheidet ein Spieler zwischen einer kooperativen Alternative (C), bei der der gemeinsame Gewinn maximiert werden kann und einer kompetitiven Alternative (D), bei der der relative Gewinn maximiert werden kann (vgl. Tabelle I).

Tabelle I. Auszahlungsmatrizen zweier Zwei-Personen-Nichtnullsummen-Spiele.

Die erste Zahl in einer der vier Zellẹn einer Auszahlungismatrix bezeichnet die Auszahlung des Spielers I (die zweite entsprechend für Spieler II), wenn dic Spieler die zugchörigen Züge der* Matrixzelle gespielt haben

PDG [17]
Spielzüge von
Spieler 1I
C

C

Spiel-

ziige

von

Spieler I

\begin{tabular}{l|l|l|}
\hline $\mathrm{C}$ & $+4,+4$ & $-5,+5$ \\
\hline $\mathrm{D}$ & $+5,-5$ & $-4,-4$ \\
\hline
\end{tabular}

MDG [12]

Spielzäge von Spieler II

C.

D

Im PDG stellt die Konsequenz (DD), daß beide Spieler sich für die kompetitive Altermative (D) entschicden haben, die spieltheoretische „Lösung" des Spieles dar - allerdings ein für beide Spieler unbefriedigendes Gleichgewicht. Man könnte von daher erwarten, daß es einige Spieler lermen, im Laufe eines Spieles, d. h. bei wiederholten Durchgängen, so etwas wie, ,Vertrauen“ und damit schließlich Kooperation zu entwickeln. Dies würde sich in der Zunahme der Konsequenz (CC), Wahl der kooperativen Alternative (C) durch beide Spieler bomerkbar machen.

Im MDG stellt die Konsequenz (CC) die rationale Lösung des Spieles dar, da hier der gemeinsam zu erreichende Gewinn der zahlenmäßig höchste überhaupt 
ist. Eine Abweichung von dieser Strategie kann erklärt werden durch das Motiv eines Spielers, den relativen Gewinn, ausgedrückt im Unterschied zwischen eigenem Gewinn und Gewinn des Spiclpartners, zu maximieren.

Sozialpsychologische Verwendungen dieser Spiele sind primär vom Interesse an der Kooperations-Kompetitions-Dichotomic dieser Spiele geleitet. Die Ceneralisierbarkeit der Ergebnisse von mit erwachsenen Vpn durchgeführten Laborexperimenten auf die Dynamik realer Konflikte ist allerdings bis heute noch umstritten [S. 16,8].

Auch die Ergebnisse der bisher mit Kindern durchgeführten spicltheoretisch konzipierten Untersuchungen lassen sich nicht problemlos in zu erwartende Entwicklıngsverläufe der Kooperativität [1] einordnen. Für den Einsatz des Forschungsparadigmas Zwei-Personen-Spiele in entwicklungspsychologischen Fragestellungen ist zunächst der Nachweis des Regelverständnisses notwendig. TEDeschI und Mitarb[17] sowie GoLD und Mitarb. [4] konnten zeigen, daß das PDG bei angemessener Darbietungsform schon von 7 -bis 8 jährigen Kindern verstanden und gespielt werden kann. Diese Autoren fanden aber keinen Effekt der Matrizen (Rapoport-Index) auf das Spielverhalten der Kinder, woraus geschlossen werden mußte, daß die Vpn nicht adäquat zwischen den subjektiven Nutzenbeträgen der Gewinne differenzicren konnten. Die Einhaltung der Präferenzordnung

$$
(\mathrm{DC})>(\mathrm{CC})>(\mathrm{DD})>(\mathrm{CD})
$$

in den subjektiven Nutzenbeträgen ist aber eine wesentliche Voraussetzung für eine sinnvolle Interpretation der Ergebnisse.

Wir wollen uns im folgenden mit der Validität der Interpretation der aus den Ergebnissen von Spielexperimenten ableitbaren Verhaltensindices (Häufigkeit von (C-Konsequenzen bzw. DD-Konsequenzen usw.) befassen, die meist in der RapoporT-Tradition ohne weitere Überprüfung als Charakterisierung kooperativer oder kompetitiver Orientierungen von Vpn aufgefaßt werden.

Die Arbeit von Pulkowski-Rebeluius [13], die den Zusammenhang zwischen Kooperativität im Spiel und soziometrischen Daten bei 12- bis 14jährigen Schulkindern untersuchte, läßt sich als ein Beitrag zur Konstruktvalidierung auffassen. Es konnten dort aber kcine Zusammenhänge aufgewiesen werden, die für die Berechtigung sozialpsychologischer Interpretation der benutzten Verhaltensindizes von Matrix-Spielen bei Kindern dieser Altersgruppe sprechen.

Auch Kobicka [9] fand bei 14jührigen Vpn keinen Zusammenhang zwischen Kooperation im Spjel und Lehrereinschätzungen über das soziale Verhalten.

McCinstock [12] konnte mit seinem MDG zwar zwischen Kindern verschiedenen Geschlechts, Alters (8, 10 und 12 Jahre) und verschiedener Subkulturen (Angloamerikaner und Mexikoamerikaner) differenzicren. Gegen seine Interpretation der mit MDG gewonnenen Daten aui der Kooperation-Kompetitions-Dimension lassen sich aber berechtigte Zweilel vorbringen:

1. Die D-Wahl kann statt Kompetition auch Minimierung des Unterschiedes ausdrücken [11]. Dies besonders auch deswegen, weil die Vpn über die eigenen kumulierten Gewinne und über die kumulierten Gewinne des Partners informiert wurden. 
2. Jüngere Kinder wählten zwar häufiger $C$ als ïltere. Befunde aus lixperimenten mit Spielen gegen die Natur [15] sowie Ergebnisse aus Lernexperimenten [18] zeigen aber, daß zum einen jüngere Kinder eher "wertorienticrtes" Verhalten zeigen - und die C-Wahl beinhaltet den höchstmöglichen Gewinn - zun anderen in ihrem Wahlverhalten ,win-stay“-Regehn bevorzugen, wodureh die größseren IÏ̈ufigkeiten von CC-outcomes erklärt werden könnten. Bei den iilteren Kindern könnte u. a. der Faktor Langweiligkeit oder die Suche nach komplexeren Wahlmustern des Gegners für die Erklärung der Zunahme von 1)-Wahlon eine Rolle spielen.

Um die Validität der üblichen spieltheoretischen Indices bei Anwendungen mit Kindern zu klären, erscheint es nötig, der Frage nachzugehen. welche andoren Verarbeitungsprozesse es im Spielgeschehen und Entscheidungsverhalten der Spielpartner in Zwei-Personen-Spielen geben könnte. Schon die möglichen Situationsauffassungen der Vpn geben uns Ifinweise darüber, ob andere Gesichtspunkte bei der Interpretation zu berücksichtigen sind.

Bei der Wahl von Zügen wäre zunächst die entscheidungstheoretische linteilung von Entscheidungen in Sicherheits-, Risiko- und Unwissenheitswahlen zu berücksichtigen. Tatsächlich handelt es sich bei der Entscheidung der $V p$ in MatrixSpielen um eine Entscheidung bei „Unwissenheit“, da nur die Auszahlungswerte der $V_{p}$ bekannt sind, nicht aber die Wahrscheinlichkeiten ihres Auftretens. LUCE und RAIFFA [10] weisen auf diese Interpretationsmöglichkeit hin und schlagen in diesem Zusammenhang die Anwendung verschiedener Entscheidungsregeln (Minimax, Laplace, Hodges-Lehmann, Minimaxregret usw.) zur Zugbestimmung vor. Die Ergebnisse von Pulkowski-Rebelluus [13] lassen sich \%. B. mit der Anwendung der Laplace-Regel erklären.

Auszuschließen ist aber nicht, daß trotzdem Wahrscheinlichkeiten für die Auszahlungen von den Kindern bzw. Vpn erlebt werden. Es ist deswegen von verschiedenen Autoren argumentiert worden, es handele sich um Risikoentscheidungen, wenn die Vpn in Zwei-Personen-Spielen wählen (z. B. [3]). Von Coovbs [2] ist daher eine Reparameterisierung des PDG in Anlehnung an seine Portfolio-Theorie vorgeschlagen worden.

Dieser Gesichtspunkt soll hitr nicht axiomatisch wie bei Coomss [2] verfolgt werden, sondern durch empirische Vergleiche. Der Gesichtspunkt Risikoentscheidung könnte gerade dann naheliegen, wenn die kognitive lahigkeit, das Spiel in allen seinen Aspekten zu verstehen und wie ein Spieltheoretiker zu analysieren, nicht vorliegt, wic wir es bei Kindern annehmen dürfen.

SAMPSON und KaRdush [14] beobachteten an ihren 7- bis 11jährigen Vpn, daß ein großer Teil $\left(71 \%\right.$ der älteren weiblichen $V_{p}$ und $75 \%$ der älturen upper-classKinder) das Risiko bei der C-Wahl betonten. Möglich ist also, dalj die Zunahme von D-Wahlen im PDG mit dem Alter nicht mit der zunehmenden kompetitiven Orientierung zusammenhängt, sondern mit einer zumehmenden Berücksichtigung und Vermeidung des Risikos in der Spielsituation.

Um das, was während einer Entscheidung wirklich erlebt bzw. beabsichtigt wịrd, von den Kindern zu erfahren, ist die Methode der Wahlbegründungen wieder- 
holt benutzt worden. Diese soll deswegen hier angewendet werden, wenngleich sich gewisse Probleme mit der Aussagekraft dieser Methode stellen. Man wird z. B. mit Verbalisierungsunfähigkeiten oder mit irrelevanten, manchmal auch als magischanimistisch einzuordnenden Antworten rechnen müssen [7]. Diese Methode erscheint aber zunächst relativ informativ, weil sie direkt an den Entscheidungsprozeß der $V_{p}$ anschließt. Andere Verfihren sind denkbar [5], setzen aber eine begründete Theorienstruktur für dic Analyse voraus, die erst im Anschlub an dicsen ersten Sehritt aufgestellt werden kann.

fï̈nstig ist es, mehrere Spiele zu verwenden, die unter unterschiedlichen Bedingungen Kooperationen oder Kompetition erfordern. Weiterhin erscheint es sinnvoll, solche Zwei-Personen-Spiele zu verwenden, die mehr als 2 Alternativen haben, damit die Zahl möglicher Begründungsaspekte erhöht wird. Dic Auszahlungsmatrizen der Spjele sollten es möglich machen, die inhaltichen Gesichtspunkte der Sicherheit und des Werts den Vpn für die Begründıngen nahezulegen. Diese Gesichtspunkte sind einerscits von Plausibilitätserwägungen angesichts der Matrizen und der Untersuchung von SAMPSON und KARDUSH [14] her, andererseits von der Forschung über die Entwicklung des Entscheidungsverhaltens in Spielen gegen die Natur her [15, 6], von Interesse. Sie konkurrieren als Gesichtspunkte mit partnerbezogenen. Unausweichlich ist es dann noch, eine ausreichende Erfahrung mit der Spielsituation vor der Befragung zuzulassen, damit die Kenntnis des Spieles ausreichend entwickelt ist. Nur so können u. U. genügend partnerbezogene Spielzüge aultreten, die dann auch entsprechend begründet werden können.

\section{Methode}

82 Vpn beiderlei Geschlechts zwischen 9 und 14 Jahren (Haupt- und Sonderschüler) spielten nach ausführlicher Einführung in die jeweilige Bedeutung der Spielmatrizen in Paaren gegeneinander drei 3-Alternativen-Zwei-Personen-Spiele (siehe Tab. II): Ein modifiziertes PDG, ein modifiziertes MDG und ein Nullsummenspiel (NSG). Die Abfolge der Spiele geschah in der gegebenen Reihenfolge.

Tabelle 1I. Auszahlungsmatrizen der betutzten Spiele (PDG, MDG und NSG) dieser Untersuchung

(Bei jedem Zugpaar bezeichnet die Zahl vor dem Lomma die Auszahlung an den Spieler 1; die Zahl hinter dem Komma die Auszahlung an Spicler 2, falls ein bestimmtes Zugpaar zustandekommi)

Spielzüge von Spieler 2

\begin{tabular}{|c|c|c|c|c|c|c|c|c|c|}
\hline & \multicolumn{3}{|c|}{ PDG } & \multicolumn{3}{|c|}{ MDG } & \multicolumn{3}{|c|}{ NSG } \\
\hline & $\Lambda$ & 13 & C & $\Lambda$ & B & C & A & B & C \\
\hline Spielzüge $\Lambda$ & 3,3 & 0,0 & $-5,5$ & 4,4 & 0,1 & $-6 ; 3$ & $3,-3$ & 0,0 & $-2,2$ \\
\hline von $\quad 13$ & 0,0 & 0,0 & 0,0 & 1,0 & 1,1 & 1,2 & 0,0 & $-3,3$ & $-1,1$ \\
\hline Spieler 1 & $5,-5$ & 0,0 & $-3,-3$ & $3,-6$ & 2,1 & $-5,-5$ & $1,-1$ & $2,-2$ & 0,0 \\
\hline
\end{tabular}

16 Z. Psychologie 187-2 
Das NSG wurde zuletzt dargeboten, um die Entstehung kompetitiver Orientierungen zu vermeiden. Es wurden 30 Züge im PDG, 35 Züge im MDG und 25 Züge im NSG gespielt, bevor die Frage gestellt wurde, warum die Vp so im letzten Zug gespielt habe. Das Spiel wurde abweichend vom sonstigen Vorgehen nicht mit einem „stooge“, sondern mit zwei naiven Partnern gespielt (d. h. beide Vpn spielten, wie es jhnen hier gut erschien), da nicht die Auswirkung der Gegner-Strategie auf das Verhalten eines Spielers untersucht werden sollte. Die Zugpaare führten zu den Auszahlungen der Tabelle II und konnten zum Einkauf von Süligkeiten beim VI verwandt werden.

Die Abbildung 1 zeigt eines der beiden verwendeten Spielgeräte. Die Wahlen erfolgten durch Betätigung eines der Taster, die den Alternativen zugcordnet waren. Die Auszahlungen für beide Spieler wurden elektronisch gesteuert nach erfolgter und beidseitiger Wahl auf jeweils einem der abgebildeten 9 Fensterchen sichtbar. Zwei Vl protokollierten nach ausführlicher standardisierter Instruktion alle Zugwahlen und die Wahlbegründungen nach den jeweils letzten Entscheidungen eines Spiels mit.

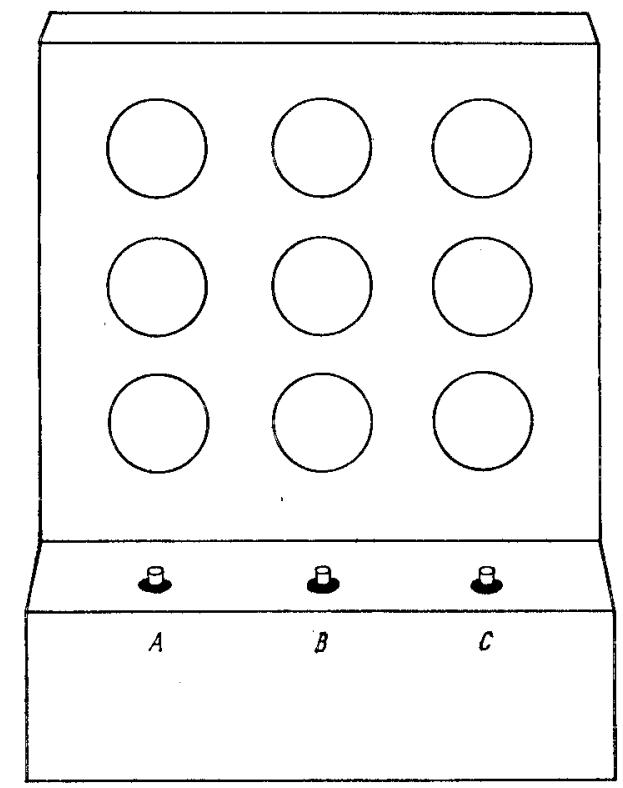

Abb. 1. Ansicht des Spielgerätes von eincm Spieler aus

Die Auszahlungen des PDG wurden unter folgenden Gesichtspunkten zusammengestellt. Für an der Maximierung des Gewinns orienticrte Spieler (wertorientierte Spicler) erscheint die Annahme berechtigt, dal der Zug C am attraktivsten ist. Dort ist sowohl der maximale Gewinn zu holen, als auch wird dieser Zug durch den Gesichtspunkt des geringeren drohenden Verlusts im Vergleich zu Zug A noch unterstützt. Zumal der wertorientierte Spieler ja überhaupt nur an dem Gewinn interessiert ist, für ihn also der Zug B überhaupt nicht in Frage kommen kann. Der Zug 
A kann für den wertoricntierten Spjeler aber' durchaus, wonn auch weniger, attraktiv sein, z. B. wenn er gar nicht auf den Verlust achtet oder nicht den höherwertigen Zug C beachtet. Für einen an Sicherheit orienticrten Spicler kann sowohl Zug B. als auch $\mathrm{Zug} C$ interessant sein: B, weil dort nichts verloren werden kann; C, weil dort weniger verloren worden kamn als boi Zug A. Das bedentet, die Auszahlungen des PDG wurdon so eingerichtet, daß für beide potenticllen mit den partnerbezogenen Sirategien konkurricrenden Begründungstypen spezifische Präfercnzordnungen ableitbar sind. Für wertorienticrte Begründer sollte am häufigsten der Zug C, dann der Zug A und am wenigsten der Zug B in Frage kommen. Für sicherheitsorienticrte Begründer mur Zug B oder Zug C ohne à priori begründbare Präferenzunterschiede.

Die Auszahlungen der anderen Spielmatrizen sind nach demselben Grundprinzip gewonnen, nur war angestrebt, dali hier andere Präferenzordnungen für die beiden Begrüundungstypen zu erwarten sind. Die Züge A und C sind im MIDG durch Wertüberlegungen begründbar. Falls cine solche Vp auch das Risiko des Verlustes miterkennt, sollte sie zwar nicht mehr so sehr vom Zug $A$ angezogen werden, weil nur in einem $F_{\text {all }}$ überhaupt ein Gewinn, dann allerdings der größtmögliche, ausgezahlt wird. Da die Einbeziehung des Verlustes hier aber nicht vollkommen mit dem Gewinnwert konkordant geht, erscheint beim MDG bezüglich der Züge A und C für wertorientierte Begrïnder keine Präferenzordnung ableitbar. Dagegen mülite hier für einen an der Sicherheit orientierten Spieler gan Attraktivität hervorstechen, da immer, wenn auch nur wenig, gewonnen wird. Die Züge A und C sollten beide gleichermaBen unattraktiv für ihn sein.

Im NSG könnten wertorientierte Spieler die Züge A und C attraktiv finden. Sobald sie zusätzlich das Risiko eines Zuges wahrnehmen, dürfte der Zug A aber an Attraktivität verlieren. Für sicherheitsorientierte Spicler dürlte im NSG nur der Zug C in Frage kommen. Es droht dort kein Verlust und ein geringer Gewinn ist wahrscheinlich.

\section{Ergebnisse}

Die Wahlbegründungen ließen sich den Kategorien der Tabelle III zuordnen.

Die Wahlbegründungshäuligkeiten in den Kategorien der Tabelle III sind in der Tabelle IV aufgeführt. Wie daraus ersichtlich, machen die auf den Spielpartner bezogenen Wahlbogründungen (Kat. IV) den geringsten Teil (durchschnittlich 8\%) aller Antworten aus. Auberdem fält in Kategorie IV auf, daß auch im NSG1 partncrorienticrte Begrïndungen bei der Wahl des spieltheoretischen Sattelpunktes (Zug C) vorkamen. Ihr Anteil ist dort allerdings am geringsten.

Weiterhin ist der Anteil von Wahlbegründungen der Kategorie $V$ in allen Spielen sehr hoch. Diese Vpn können, da sie nicht in der Lage waren, eine Begründung zu geben, in der Auswertung nicht weiter berücksichtigt werden.

1 Die Züge eines Spielers 11 in NSG warden auf Züge eines Spieler I übertragen. Die Züge A für Spieler I und Spieler II sind nämlich nach den Matrizen der Tabelle II nicht identisch. 
Tabelle III

\begin{tabular}{|c|c|c|}
\hline & Kategori: & Erklärung (Beispicl) \\
\hline 1 & $\begin{array}{l}\text { Zufallsorientierte } \\
\text { Begründungen }\end{array}$ & $\begin{array}{l}\text { Dic Verwendung von \%ufallsprozeduren wird } \\
\text { angedeutet. Das Spie! wird als Glücksspiel } \\
\text { aufgefaßt. }\end{array}$ \\
\hline II & $\begin{array}{l}\text { Wertorientierte } \\
\text { Begriundungen }\end{array}$ & $\begin{array}{l}\text { Der Versuch, den Gewinn zu maximieren, } \\
\text { dominiert in der Antwort. (,Weil ich } 5 \text { haben } \\
\text { will“.) }\end{array}$ \\
\hline III & $\begin{array}{l}\text { Sicherheitsorientierte } \\
\text { Begründungen }\end{array}$ & Das Risiko wird betont (,ist sicherer" \\
\hline IV & $\begin{array}{l}\text { Partuerorienticrte } \\
\text { Begründungen }\end{array}$ & $\begin{array}{l}\text { Das Vorhandensein des Spielpartners, Ko- } \\
\text { operation oder Kompelition wird belont. } \\
\text { („damit wir beide } 3 \text { kriegen“) }\end{array}$ \\
\hline$r$ & $\begin{array}{l}\text { Fehlende } \\
\text { Begründungen }\end{array}$ & „weiß nicht", „, nur so". \\
\hline
\end{tabular}

Tabelle IV. Häufigkeiten von Wahlbegründungen in den Kategoricu $I$ bis $V$ in $\Lambda$ bhängigkeit von der letzten Wahl in 3 Spielen

Letzte Wahl

\begin{tabular}{|c|c|c|c|c|c|c|c|c|c|c|c|c|c|}
\hline \multirow[b]{2}{*}{ Kategorie } & \multicolumn{4}{|c|}{ PDG } & \multicolumn{4}{|c|}{ MDG } & \multicolumn{4}{|c|}{ NSG } & \multirow[b]{2}{*}{ Gesamt in $\%$} \\
\hline & $A$ & B & C & $\Sigma$ & $A$ & B & $\mathrm{C}$ & $\Sigma$ & A & B & C & $\Sigma$ & \\
\hline I (Zufall) & 7 & 1 & 6 & 14 & 4 & 5 & 7 & 16 & 5 & 1 & 6 & 12 & 17 \\
\hline II (Wert) & 5 & 0 & 14 & 19 & 10 & 3 & 11 & 24 & 8 & 0 & 19 & 27 & 28 \\
\hline III (Sicherheit) & 1 & 4 & 4 & 9 & 0 & 16 & 1 & 17 & 0 & 0 & 20 & 20 & 19 \\
\hline IV (Partner) & 4 & 3 & 3 & 10 & 1. & 0 & 6 & 7 & 0 & 0 & 3 & 3 & 8 \\
\hline V (keine) & 4 & 6 & 20 & 30 & 5 & 9 & 4 & 18 & 5 & 6 & 9 & 20 & 28 \\
\hline Summe & 21 & 14 & 47 & & 20 & 33 & 29 & & 18 & 7 & 57 & & \\
\hline
\end{tabular}

Bei den Wahlhäufigkeiten im letzten Zug überwogen im PDG und NSG die $\mathrm{C}$-Wahlen. Die Abweichung von der Gleichverteilung ist mit $\chi^{2}=22.15$ und $\chi^{2}=50.57$ und jeweils 2 Freiheitsgraden hoch gesichert. Im PDG könnte dies als Vorkommen von kompetitiven Tendenzen, im NSG als Verfolgen der Minimax-Strategie interpretiert werden. Die folgende Auswertung untersucht aber die Berechtigung dieser Interpretation näher.

Zunächst können die Häufigkeiten von Vpn, die mit Wert- oder Sicherheitsbegründungen bestimmte Züge taten, mit den zuvor bei der Einrichtung der Auszahlungen der Matrizen beschriebenen Erwartungen verglichen werden. Von den WertBegründungen im PDG entfielen die meisten auf den Zug $\mathrm{C}$ und noch cinige auf den Zug $A$, was zuvor erwartet wurde. Wie erwartet fielen auch die Sicherheitsbegründungen im PDG zu gleichen Teilen auf die Züge B und C.

Im MDG entfielen Wertbegründungen etwa gleichhäufig auf die Züge A und C, nur wenige auf den Zug B. Sicherheitsbegründungen im MDG entfielen fast aus- 
schließlich auf den Zug B. Dies entspricht ziemlich genau den zuvor gehegten Erwartungen.

Auch für das NSG sind die spezifizierten Erwartungen an die Züge bestimmter Begründungstypen erfüllt. Wert-Begründer bevorzugen deutlich den Zug C, wählen aber auch den Zıg $\mathrm{A}$. Sicherheits-Bęgründer wählen nur Zug C.

Inferenzstatistisch sollen zunächst folgende Fragen untersucht werden:

- Hängt die Hänfigkeit von Begründungen in den Kategorien von der Spielart ab, oder verteilen sie sich zufällig?

- Wurden die gegebenen nicht-partnerorientierten Begründungen vermehrt zugumst'n bestimmter Kategorien gegeben, oder verteilen sie sich gemäß den Randvertcilungen zufällig?

Ein Vergleich der Randsummen der Kategorien für jedes Spiel mit der Erwartung dalüir aufgrund durchschnittlicher Häufigkeiten in der letzten Spalte (Gesant in \%) der Tabelle IV ergibt einen Wert von $\chi^{2}=13.54$, der bei vier Freiheitsgraden nicht auf dem $5 \%$-Niveau signifikant ist. Keines der Spiele führte damit in bezug auf die erste Frage zu auffiillig abweichender Verteilung der Begründıngen.

Die nicht-partnerbezogenen Begründungen in den Kategorien I bis III verteilen sich im PDG nicht von der Gleichverteilung abweichend. Die Prüfung der Abhängigkeit zwischen letzter Wahl und Begründung in Kategorie I bis III ${ }^{2}$ ergab bcim PDG cin auf dem $5 \% / 0^{\prime}$-Niveau nicht signifikantes $\chi^{2}=4.6$ bei $\mathrm{df}=2$.

Im MDG führte dagegen der gleiche statistische Test (ohne Aufteilung von Wahlen) zu ciner Prüfstatistik von $\chi^{2}=28.95$ bei $\mathrm{df}=4$, was auf dem $0.1 \%$-Niveau signifikant ist. Dentlich überrepriiscntiert waren die Häufigkeiten bei der A-Wahl in Kategoric II $\left(\chi^{2}\right.$-Anteil $\left.=10.95\right)$ und bei der B-Wahl in Kategorie III $\left(\chi^{2}\right.$-Anteil $\left.=5\right)$.

Die Öberprö̈lung dieses Zusammenhanges unterblieb für das NSG wegen der zu bohen C-Wahlenhäufigkeit, die die erwarteten Werte in den anderen Wahlen unter 5 sinken liek.

Mit der $\Lambda$ bwcichung der nicht-partnerbezogenen Begründungen von ihrer cigenen Randverteilungserwartung prült man nur, ob diese Begründungen bei verschiedenen Zü̈gen überhaupt differenzieren. Hlier war also anscheinend nur das MDG so konstruiert, dal. der Zusammenhang bei den drei Gruppen über die statistische Signifikinzgremze reichte.

Als weitere Vergleichshasis können die Zughäufigkeiten bei partnerbezogenen Begründungen dienen. Damit würde man nicht nur zeigen, daß wert-, sicherheitsund zufallsbezogene Begründungen differenzierend valide über Verarbeitungsprozesse Auskunft geben, sondern auch belegen, dab diese behaupteten und nachgewiesenen Verarbeitungsprozesse von den bei partnerbezogen auftretenden verschieden sind.

Für das PICG und das MIOG wurden deswegen die Abweichungen von der Zug-

2 Im zu geringe Jirwartungswerte zu vermejelen, geschah dies unter Aufieilung dex 5 B-Wiahlen auf die $A$-bzw. C-Wahlen. 
verteilung bei partnerbezogenen Begründungen geprüt. Für das PDG ergab ${ }^{3}$ sich bei $d f=4$ mit $\chi^{2}=25.49$ ein hochsignifikanter Wert. Im MDG mußten die Zughäufigkeiten von $A$ und $B$ zusammengelegt werden, da die Erwartung für $B$ aufgrund von partnerbezogenen Begründungen null war. Beim MDG ergab sich so bei $d f=2$ mit $\chi^{2}=126.62$ ebenfalls ein hochsignifikanter Wert. Damit wird der auch bei Betrachtung der Häufigkeiten der Tabelle IV sich aufdrängende Eindruck unterstrichen, daß die anderen Begründungsarten auf die entsprechenden Auszahlungsbedingungen in anderer Weise bezogen sind als die partnerbezogenen Begründungen.

\section{Diskussion}

Die Ergebnisse bestätigen die Vermutung, daB sich Kinder in Nicht-NullsummenSpielen überwiegend nicht partnerorienticrt, geschweige denn unter der spieltheoretischen Voraussetzung der Annahme eines rationalen Partners verhalten. Vielmehr zeigte sich, daß

- wertbezogene, sicherheitsbezogene und zufallsorientierte Begründungen übcrwiegend gegeben wurden,

- der einzelne Spielzug von verschiedenen Ipn unterschiedlich begrüundet worden kann,

- Begründungen derselben Art auf verschiedene Spiclzüge eines Spieles fallen können,

- nur bei einer bestimmten Konstruktion von Spielauszahlungen wie in MDG einc statistisch abgesicherte Beziehung zwischen verschiedencn nicht-partnerbezogenen Begründungsinhalten und vorherigem Spielzug auftrat,

- partnerbezogene Begründungen in anderer Weise mit den Zügen zusammenhingen als nicht-partnerbezogene Begründungen.

Es ist in weiteren Untersuchungen, die Matrix-Spiele bei Kindern verwenden, zu berücksichtigen, daß $\mathbf{3}$ das Verhalten von Kindern weitgehend von anderen als partnerorienticrten Jimensionen abhängt. Es kommen dann anscheinend Strategien zur Anwendung, die sich auch in Entscheidungen über Altemativen aus „Spielen gegen die Natur" nachweisen lieBen $[15,6]$.

Obwohl diese Ergebnisse wegen des geringen lorkommens von partnerorientierten Begründungen resignative Tendenzen in der Beurteilung der Verwendbarkeit von Matrix-Spielen bei Kindern einiger Autoren unterstützen [4]: zwingen sie unseres Erachtens nur zu einer differenzierteren Betrachtungsweise der Wahlen. Ansätze, die spezifisch die Entwicklung von Kooperation als Verhaltens- oder Einstellungsdimension mit diesem spieltheoretischen Instrumentarium untersuchen oder fördern wollen, erscheinen tatsächlich problematisch. Sinnvoll wäre es dagegen, das Aufkommen sozialer Orientierungen überhaupt und die Bedingungen ihres früheren oder späteren Auftretens mit diesem Instrumentarium zu untersuchen. Dabei hat allerdings die Verwendung der Wahlbegründungsmethode cinen hohen

3 Auf die sonst zu empfehlende Vermeidung von Erwartungswerten kleiner 5 beim $\chi^{2}$-Test mubte sowoll im MDG als auch im PDG verzichtet werden, sa sonst lieine statislische Prüfung möglich gewesen wäre. 
Informationswert. Abcr auch sorgfältige Auswahl von Spielen und Auszahlıng muß angeraten werden, wie die Unterschiede im MIJG und PDG zeigten.

Eine ausschliebliche Verwendung von Wahlhäufigkeiten oder durch Summierung daraus gebildeter Indizes, wie sic häulig in der Literatur vorzufinden ist, dürfte bei Kindern oder Jugendlichen in krinem $F$ all als hinreichende Informationsquelle über das psychische Geschehen in der potentiellen Interaktions-Situation ausreichen. Dabei werden nämlich, wie gezeigt, in unzulässiger Weise interindividuelle und $\mathbf{u}$. $\mathrm{U}$. auch intraindividuell unterschiedliche Prozesse durch cine hypothetische, abhängige Variable reprïsentiert. Mit anderen Worten, es ist die Bedingung der suffizienten Statistik nicht erfüllt. Diese Problematik zeigt sich sehr deutlich auch bei der 1)iskrepanz zwischen überwiegendem Vorkommen der C-Wahl im NSG, die die Verwendung der Minimax-Strategie durch die \pn anzeigen könnte, und den dazu abgegebenen Begründungen der Kinder, die überhaupt keine Hinweise auf spieltheoretische Lösungen enthielten.

In dieser Untersuchung wurden zwei „naive“ Ipn zum Spielen aufgefordert. Man könnte einwenden, bei Verwendung eincs "stooge" ließje sich mehr partnerorientiertes Verhalten boobachten. Dies läßt sich unserer Meinung nach tatsächlich aufgrund der unterschiedlichen Verstärkungsprozeduren, die verschiedene "stooge"Strategien für die allzu leicht milbinterpretierten Wahlen enthalten, erwarten. Ob damit aber eine Rechtfertigung sozialpsychologischer Interpretation dieses Verhaltens ausreichend gegeben würde, erscheint uns weiterhin zweifelhaft. Auch wenn man die Abhängigkeit des Zugverhaltens von der Partnerstrategie demonstrieren konnte, ist es für den Nachweis sozialpsychologischer Veränderungen nicht ausreichend, nur die Veränderungen von Wahlhäufigkeiten zu betrachten. Die hier vorgelegten Lirgebnisse sprechen dafür, daß eine sozialpsychologische Interpretation immer mit Hinweisen direkter Art dureh die Kinder belegt werden muß. Im übrigen empfiehlt sich diese Absicherung nicht nur, wenn Kinder in Matrix-Spielen ,interagieren", sondern immer dann, wenn nicht garantiert ist, daß die Vp das MatrixSpiel so versteht wie der VI.

\section{Zusammenfassung}

Wahlbegründungeu von 82 Kindern zur jeweils letzten Zugwahl in drei Zwei-Personen-Spielen (PDG, MDG und Nullsummen) wurden kategorisiert und auf ihren Zusammenhang mit der letzten Zugwahl hin untersucht. Es zeigle sich, daß wenige partnerorientierte Begründungen, dafür aber hauptsächlich wert- und sicherheitsorientierte Wahlbegründungen gegeben wurden. Diese hingen mit der lelzten Zugwahl zusammen.

\section{Summary}

82 children were asked to give explanations for their last choice in three Two-Person Games (PJ)G, MIX) and Zero-Sunt. These explanations were categorized and their relations to the last choices were examined. The dala showed that there are fow partner pelated but many value and security related explanillons. The explantalions depended on the last choices. 